\title{
ANÁLISE DE FATORES DEPRESSIVOS NO TRABALHO DO ENFERMEIRO NA ÁREA DE PSIQUIATRIA
}

\author{
Jair Alves Maia \\ Graduado em Enfermagem, Pós-Graduado em Didática do Ensino Superior, \\ Pós-Graduado em Saúde Pública, Mestrando em Enfermagem \\ Faculdade Israelita de Ciências da Saúde Albert Einstein São Paulo - Brasil \\ \jairalvesac@hotmail.com

\section{Leonardo Assunção Pereira} \\ Graduado em Enfermagem, Pós graduado em Unidade de Terapia Intensiva (UTI), \\ Mestrando em Enfermagem Faculdade Israelita de Ciências da Saúde Albert Einstein São Paulo - Brasil \\ Fernanda Alcântara Menezes \\ Graduada em enfermagem, Pós-graduada em Enfermagem em Centro Diagnóstico \\ Mestranda em Enfermagem Faculdade Israelita de Ciências da Saúde Albert Einstein São Paulo - Brasil
}

\begin{abstract}
Resumo:
Este estudo objetivou identificar os fatores depressivos dos enfermeiros que atuam na área de psiquiatria em um hospital de saúde mental de Rio Branco - Acre, no ano de 2014. O método utilizado foi um estudo epidemiológico, observacional, descritivo e analítico, com abordagem quantitativa. Os resultados mostram que a maioria dos enfermeiros era do sexo feminino com faixa etária de idade entre 30 a 40 anos, casados e exercendo uma jornada de trabalho dupla. O consumo de álcool, tristeza, insônia, mudança de relacionamento com a família, perda de interesse nas atividades, sentimento de desvalia, anorexia, isolamento social e uso de tranquilizantes foram identificados como os principais sintomas da depressão entre os participantes da pesquisa.
\end{abstract}

Palavras-chave: Fatores depressivos; absenteísmo; depressão; enfermeiros psiquiátricos.

\section{ANALYSIS OF DEPRESSIVE FACTORS IN NURSING WORK IN PSYCHIATRIC AREA}

\begin{abstract}
:
This study aimed to identify depressive factors of nurses working in psychiatry area in a hospital mental health of Rio Branco - Acre in the year 2014. The method used was an epidemiologic, observational, descriptive, analytical, with a quantitative approach. The results showed that most nurses were women aged between the ages of 30-40 years old, married and exerting a journey of double work, alcohol consumption, sadness, insomnia, change in relationship with family, loss of interest activities, feelings of worthlessness, anorexia, social isolation and use of tranquilizers has been identified as the main symptoms of depression among the study participants.
\end{abstract}

Keywords: Depressive factors; absenteeism; depression; psychiatric nurses. 


\section{ANÁLISIS DE LOS FACTORES DE TRABAJO DEPRESIVO DE LOS ENFERMEROS EN EL ÁREA DE PSIQUIATRÍA}

\section{Resumen:}

Este estudio tuvo como objetivo identificar los factores depresivos de las enfermeras que trabajan en el área de la psiquiatría en un hospital de salud mental de Río Branco - Acre en el año 2014. El método utilizado fue un epidemiológico, observacional, descriptivo, analítico, con un enfoque cuantitativo. Los resultados mostraron que la mayoría de las enfermeras eran mujeres de edades comprendidas entre los 30-40 años de edad, casado y ejerciendo un viaje de trabajo doble, consumo de alcohol, la tristeza, insomnio, cambios en la relación con la familia, la pérdida de interés actividades, sentimientos de inutilidad, la anorexia, el aislamiento social y el uso de tranquilizantes se ha identificado como los principales síntomas de la depresión entre los participantes del estudio.

Palabras clave: Factores depresivos; absentismo; depresión; enfermeros psiquiátricos.

\section{INTRODUÇÃO}

A depressão é uma doença que envolve alterações cerebrais, e consequentemente, físicas e sociais. Hipócrates, há 25 séculos atrás, já havia dedicado atenção e a descrito, e continua sendo um assunto em discussão pela comunidade médica e cientifica por causar sofrimento e impactar na vida social e profissional das pessoas. A depressão por ser uma doença estigmatizada, a sociedade ainda tende esconder e negar que está sendo afetada por tal problema. Devido ao estigma social, que é muito grande, os portadores da doença escusam comentários e negam que estão doentes, para evitar maiores constrangimentos (GONÇALES e MACHADO, 2015).

A saúde mental do trabalhador é um tema recorrente para quem atua na área da enfermagem. No entanto, a preocupação está voltada para os trabalhadores de outras frentes de trabalho e não direcionadas aos profissionais de enfermagem que cuidam de indivíduos com diversas doenças (FRANÇA et al., 2002).

Alguns profissionais são mais susceptíveis aos problemas de saúde mental, entre eles, o enfermeiro e o médico por interagirem, na maior parte do tempo, com pessoas que necessitam de ajuda. Inclusive, alguns fatores estressores, como clima de trabalho negativo, papéis ambíguos, e a falta de clareza em relação às tarefas a serem executadas e às expectativas, geram efeitos adversos na saúde mental desses profissionais (SILVA et al., 2012).

Uma população que merece atenção, por estar em contato com o sofrimento psíquico, 
são os profissionais de enfermagem e da área da saúde. Estes são marcados constantemente por incertezas, ansiedades, que devem ser cuidadosamente consideradas, uma vez que ao serem vivenciadas, revelam os próprios sentimentos, como também a dificuldade em manejálos. Essas situações provocam uma reação de choque entre o "bom" e o "ruim", gerada principalmente pela angústia decorrente da inadaptação do indivíduo frente a uma nova situação (GARRO et al.,2006).

A importância de conhecer a fundo a psiquiatria ocupacional se tornou fundamental por vários motivos, entre os quais destacam-se: a alta incidência de transtornos mentais entre os trabalhadores - cerca de $30 \%$ de transtornos mentais menores e $5 \%$ a $10 \%$ graves. A concessão de benefícios previdenciários por incapacidade temporária para o trabalho a profissionais da área da enfermagem, evidencia os transtornos mentais como a terceira causa dessa incapacidade, sendo superados apenas pelos acidentes e pelas doenças osteomusculares (MINISTÉRIO DA SAÚDE, 2001).

Além das condições de saúde dos pacientes, os profissionais de enfermagem passam por situações de impotência e desconforto significativo, uma vez que enfrentam dificuldades para prestar uma assistência adequada, tais como jornada prolongada de trabalho, quantidade insuficiente de profissionais, espaço físico inadequado e déficit de materiais e equipamentos. Com isso o profissional passa a vivenciar frustrações em seu cotidiano que irão contribuir para o desenvolvimento de doenças mentais (FROTA et al., 2013).

A Organização Mundial da Saúde (OMS) estima uma prevalência de aproximadamente 450 milhões de pessoas vivendo com transtornos mentais em todo o mundo, resultantes de uma complexa interação entre fatores genéticos e ambientais. Dentre os fatores ambientais que estão relacionados ao desenvolvimento de doenças mentais, o estresse, as pressões enfrentadas pelos profissionais e as frustrações que esses profissionais vivenciam a cada dia, são os mais determinantes para processos patológicos mentais (LEMOS e SOUZA, 2007).

O trabalho é uma atividade na qual aspectos físicos e psíquicos estão diretamente relacionados e podem tanto representar equilíbrio, desenvolvimento e satisfação, quanto podem causar tensão, desajuste e adoecimento do trabalhador (KIRCHHOF et al.,2009).

Estresse ocupacional é aquele oriundo do trabalho, ou seja, é um conjunto de fenômenos que se apresentam no organismo do trabalhador incapaz de enfrentar as demandas 
requeridas pela sua ocupação, podendo afetar sua saúde e seu bem-estar. Quando contínuo, favorece o aparecimento de doenças como hipertensão, úlcera, síndrome da fadiga crônica, distúrbios do sono, transtornos depressivos e síndrome de burnout. (RODRIGUES, 2013).

A consequência mais marcante do estresse profissional é a diminuição do interesse pelo trabalho, e esse desinteresse interfere nas relações e os acontecimentos deixem de ter importância e qualquer esforço pessoal pareça inútil para o profissional acometido (SKOREK et al.,2013).

Essa condição, na enfermagem, ocorre também porque as atribuições do enfermeiro demandam muita atenção, discernimento e responsabilidade, fatores que podem influenciar diretamente na saúde física e mental do trabalhador e condicionar ao surgimento do estresse ocupacional (VALERETO e ALVES, 2013).

A maioria dos estudos epidemiológicos também revelam uma relação entre os eventos de vida considerados estressantes, especialmente os negativos, o estresse crônico e o cotidiano com o início e a evolução dos transtornos depressivos. O acúmulo de eventos de vida estressantes, entre os quais se incluem, além de mortes, separações e doenças graves, início em um trabalho novo e mudanças nas condições de trabalho, parece predispor uma pessoa a episódios de depressão (SÍLVIA e JARDIM, 2011).

Questões como a produtividade, os acidentes de trabalho, o absenteísmo e os crescentes índices de sintomas psíquicos entre os trabalhadores de determinadas categorias profissionais têm sido objetos desses estudos. Dentre estas categorias profissionais, destacamse os trabalhadores da área da saúde, especialmente os atuantes em hospitais psiquiátricos, tendo em vista as inúmeras circunstâncias desgastantes presentes em seu cotidiano laboral. Assim, a insalubridade ou a penosidade do trabalho hospitalar, provém da permanente exposição a um ou mais fatores que produzam doenças ou sofrimento, decorrentes da própria natureza do trabalho e de sua organização, que são evidenciados por sinais e sintomas orgânicos e psíquicos inespecíficos (KIRCHHOF, et al., 2009).

A depressão tem sido uma das patologias que tem atingido grande parte dos funcionários públicos, inclusive os profissionais de saúde que trabalham diariamente identificando e cuidando dos problemas alheios. É uma doença caracterizada por um humor de estado deprimido, onde a pessoa sente-se triste, desmotivada, desinteressada e podendo neste processo desenvolver outras patologias mais graves (PORTO, 2006). 
A prevalência de sintomas de depressão entre os profissionais de enfermagem tem sido pouco relatada. Na literatura, existem trabalhos abordando aspectos como ansiedade, o estresse e a Síndrome de Burnout nas diversas áreas de sua atuação. Diversos autores realizaram investigações utilizando essa temática, mas não com foco central no estudo da depressão. Isso demonstra a necessidade da realização de novos estudos avaliando esse tipo de problemática direcionada à população de enfermagem (FRANCO et al., 2005).

A realização desta pesquisa justifica-se pela importância de conhecer os fatores depressivos no trabalho dos profissionais enfermeiros na área da psiquiatria. A problemática desta pesquisa foi delineada com a seguinte pergunta: "Quais os fatores que desencadeiam a depressão no profissional enfermeiro?"

Neste estudo descrevemos os fatores depressivos no trabalho do profissional de enfermagem.

\section{METODOLOGIA}

O presente estudo é de natureza epidemiológica, observacional, descritivo, analítico, com abordagem quantitativa dos dados, realizado em um hospital público de referência em psiquiatria na cidade de Rio Branco, Acre, no ano de 2014.

A pesquisa foi realizada no Hospital de Saúde Mental do Acre - HOSMAC, que dispõe de 65 leitos distribuídos em uma enfermaria feminina, uma enfermaria masculina e três leitos de emergências. Esse hospital é o único a oferecer atendimento ambulatorial, internação, urgência e emergência aos portadores de transtornos mentais do Acre, e atende pacientes de outros estados como Amazonas, Rondônia e até de outros países fronteiriços como a Bolívia e o Peru.

A amostra do estudo foi constituída por 10 profissionais enfermeiros, sendo sete do sexos feminino e três do sexo masculino que, estavam atuando na assistência aos pacientes internados na instituição no segundo semestre de 2014.

Os critérios de inclusão adotados foram: ser enfermeiro e está atuando na assistência ao paciente com transtorno mental no período da realização da pesquisa. Os critérios de exclusão adotados foram: Não aceitar em participar da pesquisa e os que estavam realizando atividades administrativas também foram excluídos da pesquisa. 
A coleta dos dados foi realizada através de uma entrevista com os enfermeiros do hospital em estudo e o instrumento utilizado foi um questionário com perguntas fechadas com a finalidade de identificar fatores depressivos no trabalho do enfermeiro.

A análise dos dados foi baseada na construção de duas tabelas: uma com os dados sociodemográficos e a outra com os dados da pesquisa com o objetivo de mostrar a presença dos fatores depressivos no exercício da profissão do enfermeiro psiquiatra.

O projeto de pesquisa foi aprovado pelo Comitê de Ética e Pesquisa (CEP) do Hospital das Clínicas de Rio Branco Acre com o parecer número 106/2011. Iniciando-se a coletas dos dados após a autorização da instituição onde foi realizada a coleta dos dados. Os enfermeiros foram orientados sobre os detalhes da pesquisa e, estando de acordo com a participação, o Termo de consentimento Livro e Esclarecido (TCLE) foi assinado pelos participantes e a pesquisa obedeceu as recomendações da resolução 466 de 2012 do Conselho Nacional de Saúde.

\section{RESULTADOS E DISCUSSÃO}

A tabela 1 apresenta os dados sócios demográficos da população em estudo, a tabela abaixo mostra que (70\%) dos entrevistados são do sexo feminino, (30\%) são do sexo masculino, (80\%) são casadas, (10\%) são solteiros, (10\%) vivem em uma união estável, (90\%) possuem idade entre 30 e 40 anos e (10\%) possui idade entre 40 e 50 anos, (90\%) são fumantes ativos e (10\%) nunca fumaram, (10\%) consomem bebida alcoólica todos os dias e (90\%) só consomem parcialmente em eventos especiais e em relação ao tempo de serviço (50\%) já trabalharam de 01 a 10 anos e (50\%) de 10 a 20 anos de serviço.

Analisando inicialmente os resultados da pesquisa observou-se que, a maioria eram do sexo feminino, estavam numa faixa etária de idade entre 30-40 anos, e se encontravam realizando uma escala dupla com quinze plantões extras por mês.

A relação do profissional com o ambiente de trabalho, promove uma série de mudanças psicológicas e comportamentais, principalmente os profissional de enfermagem que passa a conviver grande parte de sua vida com pessoas portadoras de transtorno mental. os profissionais ficam propensos a desenvolver alguns transtornos mentais ou depressivos com esse convívio a longo prazo (PAREDES et al., 2008). 
Tabela 1 - Dados Sócio demográficos, dos enfermeiros que atuam em um Hospital Psiquiátrico de Rio Branco Acre, Brasil, 2014.

\begin{tabular}{|c|c|c|}
\hline CARACTERÍSTICAS & $F(A) n=10$ & $\mathrm{~F}(\%)$ \\
\hline \multicolumn{3}{|l|}{ 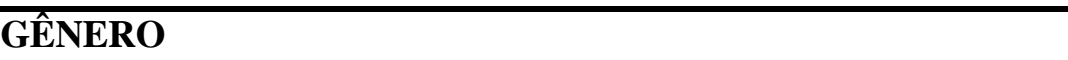 } \\
\hline Masculino & 03 & $30 \%$ \\
\hline Feminino & 07 & $70 \%$ \\
\hline \multicolumn{3}{|l|}{ ESTADO CIVIL } \\
\hline Casado & 08 & $80 \%$ \\
\hline Solteiro & 01 & $10 \%$ \\
\hline União Estável & 01 & $10 \%$ \\
\hline \multicolumn{3}{|l|}{ IDADE } \\
\hline De 30 a 40 anos & 09 & $90 \%$ \\
\hline De 40 a 50 anos & 02 & $20 \%$ \\
\hline \multicolumn{3}{|l|}{ TABAGISMO } \\
\hline Fumante ativo & 09 & $90 \%$ \\
\hline Não fumante & 01 & $10 \%$ \\
\hline Eis fumante & 00 & $00 \%$ \\
\hline \multicolumn{3}{|l|}{ ALCOOLISMO } \\
\hline Consomem ativamente & 01 & $10 \%$ \\
\hline Consomem parcialmente & 09 & $90 \%$ \\
\hline \multicolumn{3}{|l|}{$\begin{array}{l}\text { TEMPO DE } \\
\text { SERVICO }\end{array}$} \\
\hline De 01 a 10 anos & 05 & $50 \%$ \\
\hline De 10 a 20 anos & 05 & $50 \%$ \\
\hline
\end{tabular}

Fonte: Própria

O tabagismo foi considerado significativo nessa pesquisa, em relação a frequência e a quantidade de cigarros que os participantes referiram fumar durante 24 horas. $\mathrm{O}$ tabaco é a segunda droga mais consumida entre a população, devido às facilidades e estímulos para adquirir o produto e ao desconhecimento dos graves prejuízos causados à saúde dos fumantes. 


\section{ANÁLISE DE FATORES DEPRESSIVOS NO TRABALHO DO \\ ENFERMEIRO NA ÁREA DE PSIQUIATRIA}

Esse consumo tornou o hábito de fumar um comportamento socialmente aceitável, mas muito perigoso para a saúde (MAIA e MESQUITA, 2015).

A tabela 2 mostra a presença dos fatores depressivos no trabalho do enfermeiro que atua na área da psiquiatria de um hospital público de saúde mental. Dos entrevistados (100\%) referiram sintomas da depressão, (90\%) insônia (70\%) automedicação para dormir, (50\%) mudança de relacionamento com a família, (50\%) possui parentes com doença mental e apenas (10\%) faz tratamento psiquiátrico.

Tabela 2: Análise dos fatores depressivos no trabalho do enfermeiro na área de psiquiatria, em Rio Branco Acre, Brasil, 2014.

\begin{tabular}{lll}
\hline \multicolumn{1}{c}{ CARACTERÍSTICAS } & F (A) $\mathbf{n = 1 0}$ & $\mathbf{F}(\boldsymbol{\%})$ \\
\hline Sintomas da depressão & 10 & $100 \%$ \\
Sim & 00 & $00 \%$ \\
Não & & \\
Dificuldade para dormir & 09 & $90 \%$ \\
Sim & 01 & $10 \%$ \\
Não & & \\
Automedicação para dormir & 07 & $70 \%$ \\
Sim & 03 & $30 \%$ \\
Não & & \\
Mudança de relacionamento na família & & \\
Sim & 05 & $50 \%$ \\
Não & 05 & $50 \%$ \\
Familiares com transtorno mental & 05 & \\
Sim & & $50 \%$ \\
Não & 05 & $50 \%$ \\
Consulta com o médico psiquiátrico & & \\
Sim & & \\
Não & $05 \%$ \\
\hline
\end{tabular}

Fonte: Própria 
Na instituição estudada identificou-se que, os enfermeiros, na sua grande maioria, apresentavam sintomas associados à depressão como: tristeza, insônia, mudança de relacionamento com a família, perda de interesse nas atividades, sentimento de desvalia, anorexia e isolamento social.

Verificou-se que os entrevistados na sua grande maioria declararam ter uma boa relação conjugal, mas no decorrer do questionário observou-se contradições. Metade dos entrevistados referiram ter desenvolvido mudança de relacionamento com a família, o mesmo observado quando apontaram vários sintomas de depressão, apesar de anteriormente alegarem não ter nenhum problema de saúde.

A depressão é considerada um problema de saúde pública. É um dos processos patológicos com maior frequência na atenção primária médica, presente em cerca de $10 \%$ de todas as novas consultas. Afeta a população em geral, sendo altamente incapacitante e interferindo de modo decisivo e intenso na vida pessoal, profissional, social e econômica dos portadores (SILVA, 2003).

Foi observado que, $80 \%$ dos entrevistados relataram sofrer de insônia, e $70 \%$ dos mesmos fazem uso de tranquilizantes para dormir, sendo que esses medicamentos são utilizados pelos profissionais sem acompanhamento medico ou sem receita medica. Porém, a maioria deles faz uso de medicamentos para esse problema. A automedicação entre os profissionais de saúde, na maioria das vezes é motivada pela facilidade que os profissionais tem para adquirir os medicamentos que, muitas vezes não são indicados para os problemas que estão sofrendo.

A incidência de insônia neste profissionais é alta, e deteriora notavelmente a qualidade de vida das pessoas que sofrem de depressão. Apresenta consequências negativas nas atividades familiares, laborais e sociais, com isso a automedicação isolada produz um consumo nocivo e indesejável. Estas drogas, que pertencem ao grupo dos tranquilizantes menores, devido a seu amplo poder terapêutico, são motivo de abuso por profissionais de saúde (MARTINS, 1999).

Isso apresenta um quadro preocupante no caso destes profissionais. Geralmente os fármacos utilizados para insônia são de uso especial e controlado, para momentos específicos da vida, devendo ser acompanhados de um tratamento psicológico e médico para combater os fatores motivantes tanto em ordem fisiológica quanto psicológica deste problema em questão. 
Mas não é o que ocorre, e observam-se pessoas que passam anos a fio tomando dados fármacos, desenvolvendo resistência/dependência a esses, negligenciando o fator motivador da patologia, e agravando uma queda de qualidade da saúde física e mental. É uma realidade terrível, presente no cotidiano de muitos profissionais da área da saúde.

A tabela 3 apresenta os resultados acerca dos principais sintomas da depressão presentes nos servidores enfermeiros de um hospital público. Dos entrevistados (80\%) referiram ter falta de motivação, $(70 \%)$ declararam que passaram a sofrer com a falta de concentração, (60\%) declararam-se pessimistas, (90\%) declararam-se ser inseguras e (100\%) referiram ter aumentado a sensibilidade à dor.

Tabela 3 - Classificação dos sintomas depressivos investigados nos enfermeiros que estavam atuando na área da psiquiatria.

\begin{tabular}{|c|c|c|}
\hline Variável & $F(A) n=10$ & $\mathbf{F}(\%)$ \\
\hline \multicolumn{3}{|c|}{ Falta de motivação } \\
\hline Sim & 08 & $80 \%$ \\
\hline Não & 02 & $20 \%$ \\
\hline \multicolumn{3}{|c|}{ Dificuldade de concentração } \\
\hline Sim & 07 & $70 \%$ \\
\hline Não & 03 & $30 \%$ \\
\hline \multicolumn{3}{|c|}{ Pessimismo } \\
\hline Sim & 06 & $60 \%$ \\
\hline Não & 04 & $40 \%$ \\
\hline \multicolumn{3}{|c|}{ Insegurança } \\
\hline Sim & 09 & $90 \%$ \\
\hline Não & 01 & $10 \%$ \\
\hline \multicolumn{3}{|c|}{ Sensibilidade à dor } \\
\hline Sim & 10 & $100 \%$ \\
\hline Não & 00 & $00 \%$ \\
\hline
\end{tabular}

Fonte: Própria 
A depressão caracteriza-se como uma doença em que ocorrem desequilíbrios químicos dos neurotransmissores. Essas substâncias são responsáveis por transportar as informações pela rede de neurônios de nosso cérebro - incluindo as sensações de prazer, serenidade, disposição e bem estar. A depressão afeta diretamente neurotransmissores como serotonina, dopamina, noradrenalina e melatonina, que interferem nessas sensações. Quando o individuo está em depressão, há uma baixa na produção dos neurotransmissores, como a serotonina e a noradrenalina. Esses mediadores são responsáveis pela modulação da dor e também pelo equilíbrio emocional, portanto um paciente depressivo apresenta maior sensibilidade à dor (LAFER e FILHO, 1999).

\section{CONCLUSÃO}

Neste estudo foi possível identificar alguns sintomas de depressão entre os profissionais enfermeiros que participaram da pesquisa e os sintomas mais comuns foram: tristeza, insônia, mudança de relacionamento com a família, perda de interesse nas atividades, sentimento de desvalia, anorexia e isolamento social.

Considerando-se que é de fundamental importância que os enfermeiros e outros profissionais da saúde conheçam a história e os paradigmas da depressão, para que possa tornar esses conceitos explícitos, analisá-los e decidir-se por mantê-los, no seu tratamento ou transformá-los, posicionando-se com consciência.

Em situações de depressão, a abordagem médica deve ser muito cuidadosa, sendo fundamental um levantamento minucioso de dados pessoais, antecedentes de problemas psicológicos, medicamentos utilizados, além de um exame clínico completo associado à avaliação psiquiátrica e neurológica.

De acordo com a sintomatologia apresentada pelos participantes deste estudo, faz se necessário que, esses profissionais mudem a sua rotina e passem a praticar atividades físicas como caminhadas, academia e esportes e reduzam a sua carga de trabalho, para que possam melhorarem a sua qualidade de vida e seus relacionamentos com a família que é o suporte essencial na vida de qualquer ser humano.

Com base na rotina desses profissionais, propõe-se a implantação de um programa de avaliação da saúde do trabalhador dentro do próprio hospital, para que sejam atendidos os 


\section{ANÁLISE DE FATORES DEPRESSIVOS NO TRABALHO DO \\ ENFERMEIRO NA ÁREA DE PSIQUIATRIA}

profissionais do hospital e principalmente o enfermeiro que convive rotineiramente com os pacientes portadores de transtorno mental, para diagnosticar e tratar precocemente sintomas que estejam lhe afetando e que possa se agravar no decorrer do tempo, já que as doenças mentais são silenciosas.

\section{REFERÊNCIAS}

BALSAMO A.C, FELLI V.E.A. Estudo sobre os acidentes de trabalho com exposição aos líquidos corporais humanos em trabalhadores da saúde de um hospital universitário. Revista latino-americana de enfermagem. Ribeirão Preto. v.14, n.3. p. 346-53, 2003.. Disponível em: 〈http://www.scielo.br/pdf/rlae/v14n3/v14n3a07.pdf $>$ Acesso em: 27/11/2015.

BARBOSA, K.K.S.; VIEIRA, K.F.L.; ALVES, E.R.P.; VIRGÍNIO, N. A.. Sintomas depressivos e ideação suicida em enfermeiros e médicos da assistência hospitalar. Revista de Enfermagem da Universidade Federal de Santa Maria (RS) 2012 Set/Dez; 2(3): 515-522. Disponível em: <http://cascavel.ufsm.br/revistas/ojs2.2.2/index.php/reufsm/article/view/5910> Acesso em: 27/11/2015.

FRANÇA A.C.L.; RODRIGUES, A.L. Stress e Trabalho: Guia básico com uma abordagem Psicossomática. São Paulo: Atlas, 2002. 133 pp.

FRANCO, G. P.; BARROS, A. L. B. L.; NOGUEIRA - MARTINS, L. A.. Qualidade de vida e sintomas depressivos em residentes de enfermagem. Revista Latino-Americana de Enfermagem. Ribeirão Preto. v.13, n.2, p. 139-144., 2005. Disponível em: 〈http://www.scielo.br/pdf/rlae/v13n2/v13n2a02.pdf> Acesso em: $08 / 03 / 2015$.

FROTA, N, M.; BARROS, L.M.; CALDINI, L. N.; ARAÚJO, T. M.; CAETANO, J. A.. Saúde ocupacional dos profissionais de enfermagem em unidade de terapia intensiva. Revista Enfermagem em Foco. Brasília, v.4 n.2, p. 115-118, 2013; Disponível em: 〈http://revista.portalcofen.gov.br/index.php/enfermagem/article/view/525> Acesso em: 15/05/2015.

GARRO I.M.B, CAMILlO S.O, NÓBREGA M.P.S.S. Depressão em Graduandos de Enfermagem. Acta Paul Enferm. São Paulo, v.19, n.2, p.162-167, $2006 . \quad$ Disponível em: <http://www.scielo.br/pdf/ape/v19n2/a07v19n2.pdf> Acesso em: 22/08/2015.

GONÇALES, C.A.V.; MACHADO, A.L. Depressão, o mal do século: De que século? Revista de Enfermagem da Universidade Estadual do Rio de Janeiro, v.15, n.2, p. 298-304., abr/jun, 2007. Disponível em: <http://www.facenf.uerj.br/v15n2/v15n2a22.pdf> Acesso em: 20/09/2015.

JARDIM S. Depressão e trabalho: ruptura de laço social. Revista brasileira de saúde ocupacional. São Paulo, v.36, n.123, p.84-92, 2011. Disponível em: 〈http://www.scielo.br/pdf/rbso/v36n123/a08v36n123.pdf $>$ Acesso em: $20 / 08 / 2015$.

KIRCHHOF, A.L.C. Condições de trabalho e características sociodemográficas relacionadas à presença de distúrbios psíquicos menores em trabalhadores de enfermagem. Texto Contexto Enfermagem. Florianópolis. v.18, n. 2., p. 215-223, abr-jun, 2009. Disponível em: 〈http://www.scielo.br/pdf/tce/v18n2/03.pdf > Acesso em: $21 / 04 / 2015$

LAFER, B; FILHO, H.P.V. Genética e fisiopatologia dos transtornos depressivos. Revista Brasileira de Psiquiatria, São Paulo (SP) 1999; vol. 21. Disponível em: <> Acesso em:

LEMOS, S.;SOUZA, M.G.. Preparo do enfermeiro da atenção básica para a saúde mental. Arquivo Ciências da Saúde. São José do Rio Preto. v. 14, n.4, p. 198-202, out-dez, 2007. Disponível em: 〈http://repositorioracs.famerp.br/racs_ol/vol-14-4/ID227.pdf> Acesso em: 03/10/2015 
MAIA J.A.; MESQUITA R.O. Experiências e percepções de mães usuárias de drogas atendidas em uma unidade de saúde da atenção primária. Revista Científica da Faculdade de Educação e Meio Ambiente. Ariquemes, v.6, n.1, p.1-13, jan-jun, 2015. Disponível em: <http://www.faema.edu.br/revistas/index.php/RevistaFAEMA/article/view/275> Acesso em: 17/08/2015

MARTINS, L. M. M. Assistência de enfermagem a pacientes com desordem bipolar e sentimentos da estudante de enfermagem: estudo de caso. Rev. esc. enf. USP. São Paulo, v.33, n.4, 1999. Disponível em: $<$ http://www.ee.usp.br/reeusp/upload/pdf/478.pdf > Acesso em:15/12/2014.

MINISTÉRIO DA SAÚDE. O campo da saúde do trabalhador e o papel dos profissionais de saúde na atenção à saúde dos trabalhadores. In: Dias E.C.D., org. Doenças relacionadas ao trabalho: manual de procedimentos para os serviços de saúde. Brasília: Editora MS, 2001, p. 17-26. Disponível em: <http://bvsms.saude.gov.br/bvs/publicacoes/doencas_relacionadas_trabalho1.pdf > Acesso em: 05/03/2015.

PAREDES, N.P; MIASSO, A.I; TIRAPELLI, C.R.. Consumo de benzodiazepinos sem prescrição médica entre estudantes do primeiro ano da Escola de Enfermagem da Universidade de Guayaquil, Equador. Revista latinoamericana de enfermagem. Ribeirão Preto, v.16 (especial), mai-jun, 2008. Disponível em: $\langle$ http://www.scielo.br/pdf/rlae/v16nspe/pt_21.pdf $>$ Acesso em: 15/12/2014.

PORTO, J. A. Depressão: Conceito e Diagnostico. Revista brasileira de psiquiatra. São Paulo, v.21, n.1, 2006. Disponível em: <http://www.scielo.br/pdf/rbp/v21s1/v21s1a03.pdf> Acesso em: 15/12/2014.

RODRIGUES, C.S, DENISE. Modelo Demanda-Controle e estresse ocupacional entre profissionais de enfermagem: revisão integrativa. Revista brasileira de enfermagem. Brasília, v.66, n.5, p.779-788, set-out, 2013. Disponível em: 〈http://www.scielo.br/pdf/reben/v66n5/20.pdf> Acesso em: 16/12/2014.

SILVA, M.C. F; FUREGATO, A.R. F; JÚNIOR, M.L.C. Depressão: Pontos de vista e conhecimento de enfermeiros da Rede Básica de Saúde. Revista latino-americana de enfermagem. Ribeirão Preto, n.11, p.7-13, 2003. Disponível em: <http://www.revistas.usp.br/rlae/article/view/1728/1773> Acesso em: 26/07/2015.

SKOREK, J.; SOUZA, R. A.; BEZERA, R.M.. Síndrome de burnout em enfermeiros atuantes em uma Unidade de Terapia Intensiva. Revista de enfermagem da Universidade Federal de Pernambuco. Recife. v. 8 n.7., p. 1841-1847, $\quad$ jul. $2014 . \quad$ Disponível em: <http://www.revista.ufpe.br/revistaenfermagem/index.php/revista/article/viewArticle/3146> $>$ Acesso em:27/11/2015.

VALERETO F.A., ALVES D.F.. Fatores desencadeantes do estresse ocupacional e da síndrome de burnout em enfermeiros. Revista Saúde Física \& Mental- UNIABEU. Rio de Janeiro, v.3, n.2, p.: 1-11, ago-dez,2013. Disponível em: <http://www.uniabeu.edu.br/publica/index.php/SFM/article/view/1192/1038> Acesso em: 22/08/2015 